\title{
Real-time continuous estimation of dross attachment in the laser cutting process based on process emission images
}

Cite as: J. Laser Appl. 32, 042016 (2020); https://doi.org/10.2351/7.0000145

Submitted: 30 April 2020 . Accepted: 13 October 2020 . Published Online: 10 November 2020

(D) Matteo Pacher, Luca Franceschetti, (D) Silvia C. Strada, (iD) Mara Tanelli, (iD) Sergio M. Savaresi, and (D) Barbara Previtali

\section{ARTICLES YOU MAY BE INTERESTED IN}

Geometry and absorptance of the cutting fronts during laser beam cutting Journal of Laser Applications 32, 032015 (2020); https://doi.org/10.2351/7.0000024

Anticipating heat accumulation in laser oxygen cutting of thick metal plates Journal of Laser Applications 32, 022018 (2020); https://doi.org/10.2351/7.0000052

Ultralow duty cycle chopper instigated low power continuous wave laser assisted synthesis of silver nanoparticles: A novel approach

Journal of Laser Applications 32, 042017 (2020); https://doi.org/10.2351/7.0000215

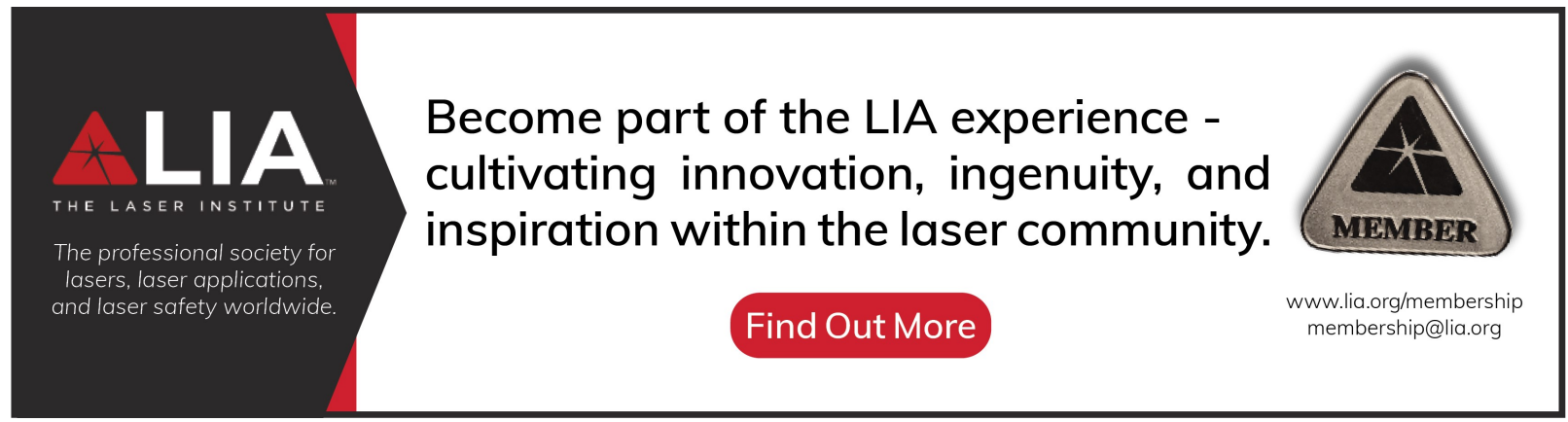




\title{
Real-time continuous estimation of dross attachment in the laser cutting process based on process emission images
}

Cite as: J. Laser Appl. 32, 042016 (2020); doi: 10.2351/7.0000145

Submitted: 30 April 2020 - Accepted: 13 October 2020 .

Published Online: 10 November 2020

Matteo Pacher, ${ }^{1,2, a)}$ (D) Luca Franceschetti, ${ }^{3}$ Silvia C. Strada, ${ }^{3}$ (D) Mara Tanelli, ${ }^{3,4}$ (D) Sergio M. Savaresi, ${ }^{3}$ (D) and Barbara Previtali ${ }^{1}$ iD

\author{
AFFILIATIONS \\ ${ }^{1}$ Dipartimento di Meccanica, Politecnico di Milano, Via La Masa 1, 20156 Milano, Italy \\ ${ }^{2}$ Adige S.P.A., BLM GROUP, Via per Barco 11, 38056 Levico Terme (TN), Italy \\ ${ }^{3}$ Dipartimento di Elettronica, Informazione e Bioingegneria, Politecnico di Milano, via G. Ponzio 34/5, 20133 Milano, Italy \\ ${ }^{4}$ Istituto di Elettronica e Ingegneria dell'Informazione e delle Telecomunicazioni-IEIIT CNR, Corso Duca degli Abruzzi 24, \\ 10129 Torino, Italy
}

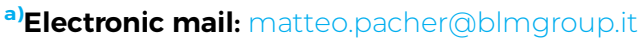

\begin{abstract}
Laser cutting of metals has become the reference manufacturing technology in sheet metal working thanks to the flexibility and the increased productivity it offers when compared with other competitive technologies. Considering, in particular, the fusion-cutting mode, i.e., when nitrogen is used as an assisting gas, different aspects contribute to the process quality among which dross attachment plays the most important role. To cope with the related time-dependent deterioration of the process quality and to obtain an online adaptation of the process parameters for different working conditions, a closed-loop dross regulation system is needed. To realize it, a reliable, continuous, and accurate estimation of the dross is mandatory. This work focuses on this challenging problem, presenting and comparing different approaches to estimate the dross attachment based on the process emission collected by a coaxial camera. Specifically, a method which relies on the accurate analysis of the process emissions for determining an effective classification method is compared with a deep-learning approach based on convolutional neural networks. The obtained results, validated in real experimental conditions, confirm the possibility to accurately estimate the presence of significant dross attachment in real-time and open the way to the design of a closed-loop control algorithm for the real-time regulation of the dross attachment formation and consequently of the process quality.
\end{abstract}

Key words: laser cutting, process monitoring, camera monitoring, dross attachment, real-time estimation, real-time

Published under license by Laser Institute of America. https://doi.org/10.2351/7.0000145

\section{INTRODUCTION AND BACKGROUND}

For many applications and particularly for cutting metal sheets and thin tubes, laser cutting has become the reference technology thanks to its flexibility and the gain of productivity it permits compared to other competitive technologies, such as abrasive water jet cutting and electrical discharge machining. Furthermore, considering the laser-based manufacturing processes, laser cutting has indeed a prevalent position with revenues that amount to approximately $41 \%$ of the total laser sources' market. ${ }^{1}$ Considering laser cutting of metals, two cutting modes exist, depending on the assisting gas used: oxidation cutting, i.e., when oxygen is used, and fusion cutting, i.e., when nitrogen is used. ${ }^{2-4}$ Both oxidation and fusion cutting modes are widely adopted in industrial applications; ${ }^{5,6}$ however, fusion cutting is currently replacing oxidation cutting in many cases thanks to the increasing laser power availability and its advantages. Indeed, fusion cutting offers a high productivity gain for low-thick materials, and it permits to cut a wider range of metallic alloys, even if it yields increased roughness, dross attachment and requires a higher laser power. As a consequence, it is of utmost importance to be able to 
assess the process quality of the fusion-cutting mode. This work aims at doing this with an automatic online process that allows quantifying the dross attachment level starting from the analysis of the process images, obtained with a high-quality camera.

The quality of the laser cutting process is traditionally determined by considering different quantities, namely, dross attachment, kerf width, surface roughness, heat affected zone, and the presence of burns on the cut edge. ${ }^{2}$ However, these features do not have equal relevance, and it has been found that the dross attachment is indeed the most influential output parameter. ${ }^{7,8}$ Accordingly, this study focuses on dross attachment only and proposes to estimate a continuous and quantitative measurement of such quantity that is to be estimated online from process observation.

Nowadays, the optimal process parameters are usually found via empirical modeling. Thus, the optimal process parameters are mostly constant, and they are usually achieved in well controlled and standard conditions. To cope with numerous uncontrollable factors that affect real industrial environments, e.g., different material properties, worn lenses, and/or machine parts, process parameter values are usually set in a precautionary way. As a consequence, a trade-off between quality and productivity arise, as conservative parameter choices are safer but decrease productivity. Thus, a margin either in productivity or in achievable quality could be exploited for specific production needs. In this framework, the development of an adaptive control logic that adapts the process parameters according to a specific quality requirement would increase both reliability and productivity of the process by overcoming the limitations of precautionary constraints. To this end, the development of a real-time, continuous, and accurate quality estimation system becomes the enabling and fundamental prerequisite.

In the literature, several contributions address the monitoring of the laser cutting process with different techniques and sensors, see, e.g., , Refs. 9-18. Considering the fusion-cutting mode, a group from the University of the Applied Science of Aschaffenburg guided by Adelmann presented some contributions to detect loss-of-cut conditions ${ }^{19-22}$ and to detect dross attachment ${ }^{23}$ based on photodiode sensors. In the latter contribution, ${ }^{23}$ the authors show how dross attachment is correlated with the variation of the photodiode signal. However, the level of dross produced in the experiments is unrealistically high.

The use of cameras has become, in the last two decades, a viable alternative to photodiode-based monitoring. Initially, the shape of the spark cone has been studied and correlated to different processing conditions. ${ }^{24-27}$ This technique proved effective for quality estimation; however, the required setup is directiondependent and does not permit industrialization. To overcome this issue, coaxial setups were proposed. Hafterkamp et al. ${ }^{28}$ and Poprawe $^{29}$ depicted the potentials of coaxial monitoring of the laser irradiated zone. Hafterkamp ${ }^{28}$ focused on the analysis of practical issues such as the detection of the misalignment between the nozzle and the laser beam, measurement of the kerf width, and detection of irregular power distribution. Poprawe ${ }^{29}$ compared the results of coaxial camera monitoring with process simulations showing the relationship between process emission and cutting quality. The state-of-the-art of coaxial camera-based monitoring is represented by the contributions of Duflou et al. ${ }^{30,31}$ In these studies, different quality features of the oxidation cutting mode were detected from process emission images and a closed-loop algorithm was developed for the automatic optimization of process parameters in the oxidation cutting mode. The same research group also developed a plasma-prevention control algorithm based on the same hardware. ${ }^{32}$ Considering the two sources of information, coaxial camera monitoring has been selected for the use in the present work thanks to the availability of spatially resolved information. In fact, photodiode-based monitoring could be, in principle, used to detect extreme dross attachment conditions, as in Ref. 23, but it hardly could be used to continuously estimate the amount of dross that is produced.

This work addresses the problem of online estimation of dross attachment by means of indirect methods based on the process emission collected by the coaxial camera. More specifically, the main goal is to produce an online continuous signal to be used for control purposes in the field of fusion laser cutting. Process emission images have been collected using a high-speed camera filtered in the near infrared region; images have been processed using two different approaches: a machine-learning (ML) algorithm where the features of interest are derived from user experience and a convolutional neural network $(\mathrm{CNN})$ algorithm that autonomously maps images onto the estimated dross attachment. A final comparison between the two approaches is presented considering both accuracy and computational performance.

The paper is organized as follows. Section II describes the experimental setup and shows the different types of dross that have been obtained throughout the experiments. In Sec. III, the dross attachment measuring procedure is explained as well as the variables that have been used in the estimation algorithms. Section IV details the various step of the machine-learning algorithm, whereas Sec. $V$ outlines the structure of the CNN algorithm and the step to train and test its performance. The comparison and discussion of the two algorithms are given in Sec. VI.

\section{EXPERIMENTAL SETUP}

A industrial laser cutting machine has been used in the experiments. It is endowed with a fiber laser source that can deliver up to $6 \mathrm{~kW}$ of power and has a fiber core diameter of $d_{\text {core }}=100 \mu \mathrm{m}$. The machine mounts a standard cutting head that has been customized for monitoring purposes (see Fig. 1). The parameters of the optical chain are reported in Table I. The monitoring architecture is composed of a camera with appropriate filters and mounted coaxial to the laser beam. The selected camera sensor is an industrial CMOS camera based on Si photodetectors with a sensitivity between 350 and $1000 \mathrm{~nm}$. Sensor size and pixel size are $1280 \times 1024$ pixels and $4.8 \times 4.8 \mu \mathrm{m}^{2}$, respectively; the camera permits to adjust the region of interest that influences both the field of view and temporal resolution. The camera lens and the number of pixels were selected to have a field of view of $2 \times 2 \mathrm{~mm}^{2}$ and a spatial resolution of $9.6 \mu \mathrm{m} /$ pixel. Considering the performance of the camera, monochrome images of size $210 \times 210$ pixels were acquired at $1500 \mathrm{fps}$; furthermore, based on the existing scientific literature and other studies, ${ }^{30,33,34}$ a near infrared wavelength range was selected and a bandpass filter centered at $750 \mathrm{~nm}$ was introduced in the optical chain. The selected wavelength is lower than the values reported in 


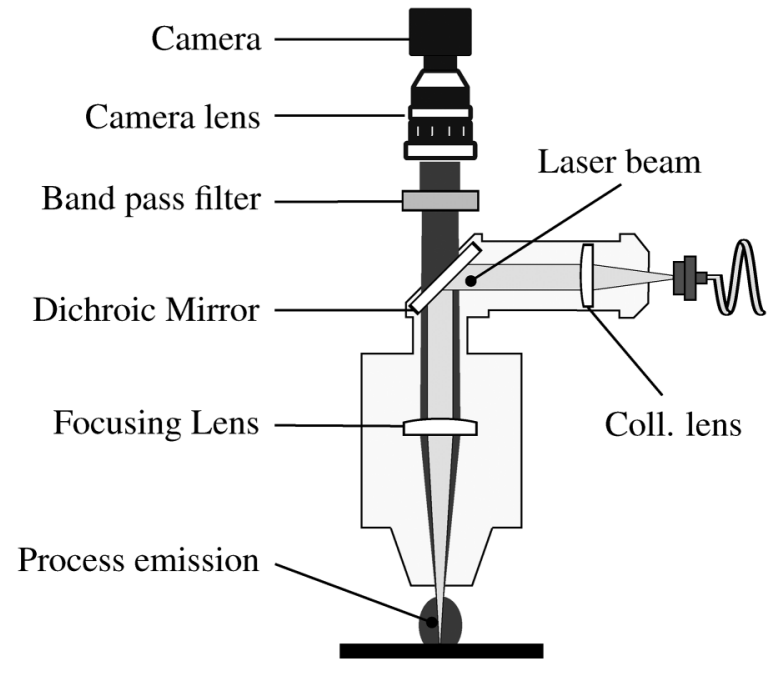

FIG. 1. Implementation of the monitoring architecture on the laser cutting head.

relevant literature studies; ${ }^{16,17,30}$ this choice was made considering the limitations introduced by the optical elements of the cutting head and the sensitivity region of the sensor. Eventually, the developed solution permitted to enhance the temporal resolution of two orders of magnitude with respect to other studies ${ }^{26,29,30}$ and provided adequate information for the purpose of the presented paper. A microcontroller was used to acquire the process parameters (e.g., cutting speed, laser power, etc.) at a sample rate of $10 \mathrm{kHz}$ and for producing a transistor-transistor logic (TTL) signal to be used as trigger for the image acquisition; this eventually permitted to perfectly synchronize images and process inputs. To measure the dross attachment, a microscope having a resolution equal to $5 \mu \mathrm{m}$ and field of view of $23 \times 17 \mathrm{~mm}^{2}$ has been used. The specific products used to build the experimental setup are reported in the Appendix.

To study and monitor the dross attachment formation, different experiments have been carried out where the goal was to produce a large quality variation in order to collect both dross-free (good-quality) and high-dross (low-quality) cuts. As can be found in the literature, ${ }^{2,35,36}$ different types of dross attachment can be obtained for different combinations of the process parameters. Indeed, considering that the laser power is generally fixed at the maximum installed power and that the standoff distance and the

TABLE I. Parameters of the laser optical chain.

\begin{tabular}{cc}
\hline \hline Laser wavelength, $\lambda$ & $1070 \mathrm{~nm}$ \\
Max. laser power, $P_{\max }$ & $6 \mathrm{~kW}$ \\
Beam quality factor, $M^{2}$ & 11.7 \\
Fiber core diameter, $d_{\text {core }}$ & $100 \mu \mathrm{m}$ \\
Collimation lens, $f_{c}$ & $100 \mathrm{~mm}$ \\
Processing lens, $f_{p}$ & $200 \mathrm{~mm}$ \\
Nominal waist diameter, $d_{0}$ & $200 \mu \mathrm{m}$ \\
\hline \hline
\end{tabular}

nozzle size are kept constant, the dross attachment is formed by varying the cutting speed, the focal position, and the gas pressure values. $^{2}$ In this framework, it is known that dross attachment forms at low and high speed, respectively, and that there exists a speed value defined as optimal at which the dross is minimized and/or not present. ${ }^{35}$ During the experiments, a standard squared geometry with a side length of $45 \mathrm{~mm}$ was selected and two materials, namely, stainless steel X5CrNi18-10, and mild steel S235JR were cut for thicknesses ranging from 3 to $10 \mathrm{~mm}$. The laser power was kept constant at $6 \mathrm{~kW}$, whereas cutting speed, gas pressure, and focal position were varied to obtain different levels of dross attachment. The experiments were not designed in standard factorial plans due to the strong correlation between process parameters (e.g., cutting speed and focal position); during the experiments, the process parameters were varied in an unstructured manner, testing different material and thickness combinations following the known laser cutting trends for producing variable levels of dross. For reproducibility, the range of variation of the process parameters is reported in Table II; the standoff distance was set to $0.5 \mathrm{~mm}$; some meaningful examples showing the achieved variability are discussed in what follows.

Considering different processing conditions, a set of different dross types were obtained. Figure 2 shows the dross attachment due to a variation of the cutting speed. It is observed that dross due to low speed appears as a remelted and homogeneous layer strongly attached to the material; furthermore, the remelted region extends in vertical direction and it is relatively wide. Its removal is hard and requires proper postprocessing steps. High-speed dross, on the other hand, is characterized by isolated droplets that discontinuously are attached at the bottom surface. The phenomenon is almost completely localized at the very bottom surface and the droplets are usually not hard to remove.

In addition to cutting speed, focal position plays a relevant role in determining the type of dross that is produced. In Fig. 3, an example of different types of dross due to different focal positions is depicted. One may observe that decreasing the focal position helps in reducing dross attachment, and that two kinds of dross are completely different, making them classifiable as low-speed and high-speed dross, respectively. Finally, if the focal position is too large (e.g., $>T_{n} / 2$ ), dross formation cannot be avoided and it

TABLE II. Ranges of variation of the process parameters.

\begin{tabular}{lccccc}
\hline \hline $\begin{array}{l}T_{n}^{\mathrm{a}} \\
(\mathrm{mm})\end{array}$ & $\begin{array}{c}P^{\mathrm{b}} \\
(\mathrm{kW})\end{array}$ & $\begin{array}{c}v^{\mathrm{c}} \\
\left(\mathrm{m} \mathrm{min}^{-1}\right)\end{array}$ & $\begin{array}{c}p^{\mathrm{d}} \\
(\mathrm{bar})\end{array}$ & $\begin{array}{c}f^{\mathrm{e}} \\
(\mathrm{mm})\end{array}$ & $\begin{array}{c}d_{\text {nozzle }} \\
(\mathrm{mm})\end{array}$ \\
\hline 3 & 6 & $6.0-9.7$ & $8-12$ & -1.0 to -2.8 & 1.8 \\
5 & 6 & $4.0-6.5$ & $12-20$ & -2.3 to -4.3 & 2.3 \\
8 & 6 & $2.0-3.5$ & $12-25$ & -6.0 to -7.5 & 2.5 \\
10 & 6 & $0.8-1.4$ & $16-25$ & -8.5 to -9.0 & 3.0 \\
\hline \hline
\end{tabular}

${ }^{a}$ Nominal plate thickness.

${ }^{\mathrm{b}}$ Laser power.

${ }^{\mathrm{c} C u t t i n g}$ speed.

${ }^{\mathrm{d}}$ Gas pressure.

'Focal position.

${ }^{\mathrm{f}}$ Nozzle outer diameter. 


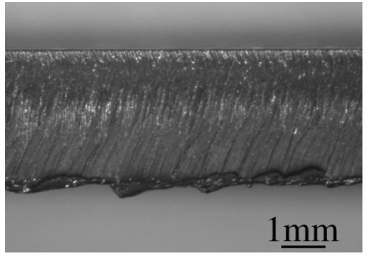

(a) Low-speed dross $\left(v=3.2 \mathrm{mmin}^{-1}\right)$.

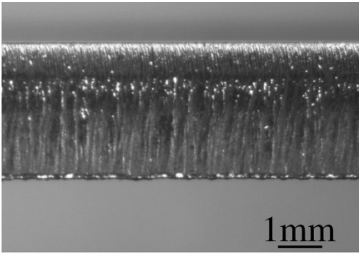

(b) Best quality $\left(v=7.0 \mathrm{mmin}^{-1}\right)$.

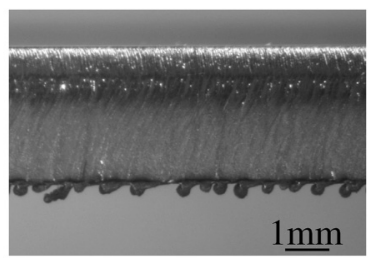

(c) High-speed dross $\left(v=9.7 \mathrm{mmin}^{-1}\right)$.

FIG. 2. Cut edges for different process conditions. Low-speed dross (a) and high-speed dross (c) are shown together with best quality cuts (b); other parameters: $\mathrm{X} 5 \mathrm{CrNi} 18-10, T_{n}=3 \mathrm{~mm}, f=-2.8 \mathrm{~mm}, P=6 \mathrm{~kW}$, and $p=12$ bar.

results in a hard and continuous layer as occurring for the lowspeed dross.

To conclude the analysis, typical dross types for different materials are depicted in Fig. 4. It is observed that for mild steel, dross attachment appears continuous and smooth. For stainless steel, instead, dross attachment is generally discontinuous and sharp. This can be explained considering the thermal conductivity and oxidation properties of the two materials: mild steel has a higher thermal conductivity compared to stainless steel; typical reference values for the thermal conductivity are $40-45 \mathrm{~W} \mathrm{~m}^{-1} \mathrm{~K}^{-1}$ for mild steel and $14-$ $16 \mathrm{~W} \mathrm{~m}^{-1} \mathrm{~K}^{-1}$ for stainless steel. Accordingly, the molten material cools down more quickly and likely keeps attached to the bottom surface. Furthermore, due to oxidation, the droplets of molten material are smooth and rounded for mild steel.

\section{DROSS ATTACHMENT MEASUREMENT PROCEDURE}

A quantitative measurement of dross attachment is fundamental for the design of an online estimation algorithm, as it allows

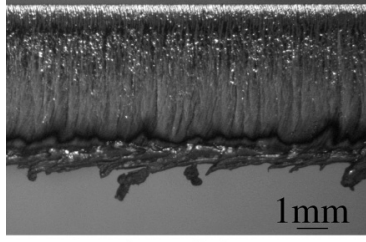

(a) $f=-2.3 \mathrm{~mm}$.

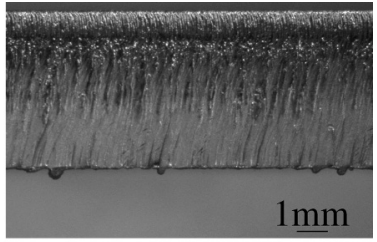

(b) $f=-4.3 \mathrm{~mm}$.
FIG. 3. Dross types for high (a) and low(b) focal positions; other parameters: X5CrNi18-10, $T_{n}=5 \mathrm{~mm}, P=6 \mathrm{~kW}, v=6.4 \mathrm{~m} \mathrm{~min}^{-1}$, and $p=12$ bar.

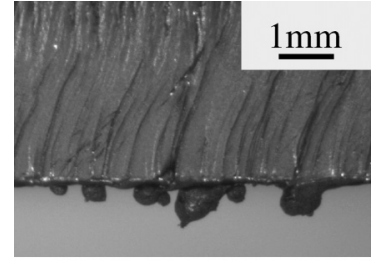

(a) Stainless steel.

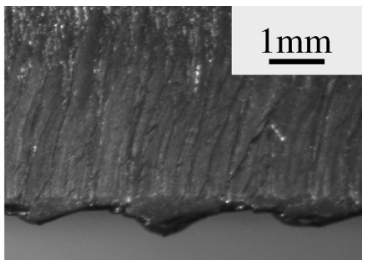

(b) Mild steel.
FIG. 4. Dross attachment types for stainless steel (a) and mild steel (b); other parameters: $T_{n}=5 \mathrm{~mm}, v=6.2 \mathrm{~m} \mathrm{~min}^{-1}, f=-4.0 \mathrm{~mm}, P=6 \mathrm{~kW}$, and $p=12$ bar.

both to train the model and to validate its performance in the testing phase. In fact, when developing the observer, there is the need to quantitatively map features obtained from the monitoring chain to a variable representing the true amount of dross produced. To construct this measurement, the whole specimen's profile is considered for dross measurements to allow postprocessing and eventually averaging of the local dross profile. Due to the reduced field of view of the microscope, three pictures with partial overlapping of each side are collected. Each specimen's side profile is then reconstructed through image stitching.

Once the pictures have been collected, the implemented image analysis tool performs the following steps:

(1) full side reconstruction through image stitching; ${ }^{3}$

(2) extraction of the top surface boundary for misalignment compensation;

(3) extraction of left and right boundaries; and

(4) extraction of the bottom profile.

Steps between 2 and 4 rely on the same algorithm for boundary detection via gradient analysis. ${ }^{38}$ The algorithm flow is displayed in Fig. 5. According to the measurement procedure, the thickness profile $T(s)$ in Fig. 5 is expressed as a function of the horizontal abscissa $s$ in the spatial domain. Let us consider a discrete-time
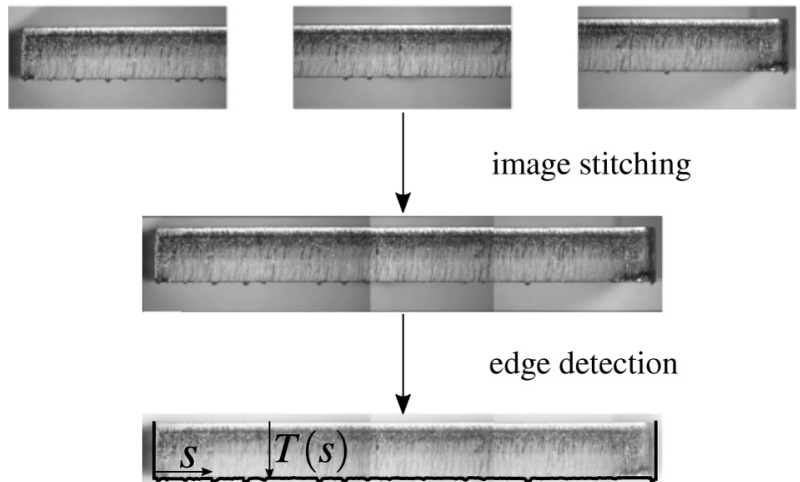

FIG. 5. Steps of the image analysis algorithm used for dross attachment measurements. $s$ is the horizontal coordinate for each side and $T$ represents the thickness of the specimens including dross attachment. 
abscissa $k=i \Delta t, i \in \mathbb{N}$, where $k$ is the discrete-time abscissa and $\Delta t$ is the sampling period. The displacement as a function of time: thus, the variable $s(k)$ is computed by integrating the feed rate, and the resulting variable is then used to express the thickness profile in the time domain as

$$
T(s)=T(s(k))=T(k) .
$$

The thickness $T$ is made of both the nominal thickness of the sheet, $T_{n}$, and the dross attachment. As the nominal thickness is known, the dross attachment profile, $h(k)$, can be computed as

$$
h(k)=T(k)-T_{n} .
$$

Finally, to emphasize the dross droplets with respect to the thickness baseline, the dross profile has been squared, yielding

$$
h^{2}(k)=\left(T(k)-T_{n}\right)^{2} .
$$

Starting from the dross measurement, a dimensionless quantity for online estimation is defined, which may eventually become the system output employed for future control purposes. This quantity has the advantage to describe the dross attachment in a more general way with respect to the traditional measurement of the dross height. In fact, dross attachment is not only characterized by the droplets height but their rate of appearance is also important for the attribution of quality. On the one hand, it has been found that small droplets are usually tolerated by technicians and customers. On the other hand, when the droplets are almost continuous [see, e.g., Fig. 2(c)], the perceived level of dross is significant even if the height of the droplets is small. To capture both aspects in the system output, two steps are carried out.

(1) investigation of the relationship between the squared dross height $h^{2}(k)$ and the judgment of technicians to determine a significant height value for which the droplets are considered unacceptable and

(2) definition of a variable to be estimated which is representative of the cut quality, and it is able to capture the rate of appearance of significant droplets together with their overall height.

To set the significant dross height according to the judgment of skilled technicians, 100 specimens of different materials and with different thicknesses were analyzed. For each specimen, a binary indicator of dross (dross presence or not) was obtained, and considering these judgments, a threshold value, $h_{0}^{2}$, for the quantity $h^{2}(k)$ was determined. In Fig. 6, two examples of dross-free and mid-dross cuts are shown together with the resulting threshold value. Once the threshold value has been set, the quantity $h^{2}(k)$ can be transformed to a binary signal indicating the appearance of significant droplets, i.e.,

$$
h_{t h}^{2}(k)= \begin{cases}1 & h^{2}(k)>h_{0}^{2}, \quad k \geq 0, \\ 0 & \text { otherwise, }\end{cases}
$$

where $h_{0}^{2}=0.03 \mathrm{~mm}^{2}$. The quantity $h_{t h}^{2}(k)$ is dimensionless and can be useful to train and test classification algorithms as it will be addressed in Sec. V. However, this quantity is not continuous and,
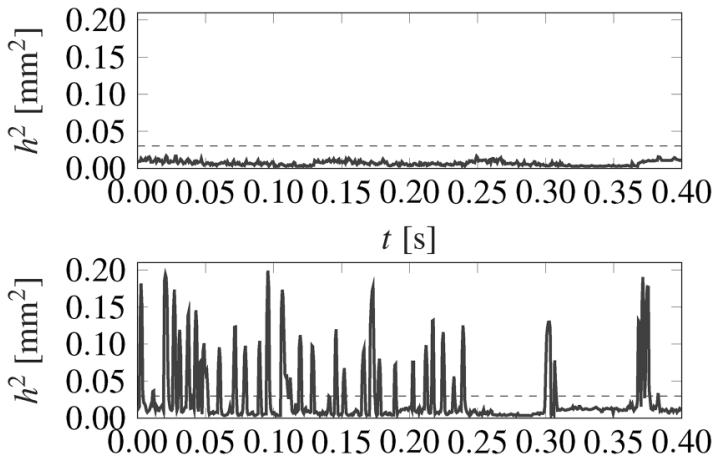

$t[\mathrm{~s}]$

FIG. 6. Comparison between a dross-free cut and a mid-dross cut. The baseline for droplets significance is also shown.

therefore, it will not permit a continuous regulation of the dross amount to a desired value. The system output is finally defined as the moving average of $h_{t h}^{2}(k)$ and computed as

$$
y(k)=\frac{1}{\tau+1} \sum_{j=0}^{\tau} h_{t h}^{2}(k-j),
$$

where $\tau$ is the size of the look-back window. The signal $y(k)$ is the bounded within the interval $[0,1]$. It indicates how frequent is the appearance of significant dross droplets (i.e., above the threshold $h_{0}^{2}$ ) within a time interval of $\tau \Delta t$. As a result, this quantity puts together the dynamic behavior of dross attachment with its significance in terms of magnitude. This signal, $y(k)$, constitutes the final quantitative measure of dross attachment that is estimated by the two algorithms and used to evaluate their performance.

\section{DROSS ATTACHMENT ESTIMATION BY MEANS OF A MACHINE-LEARNING ALGORITHM}

The aim of this section is to design an online estimate, $\hat{y}(k)$, of the previously defined system output, $y(k)$, based on the process emission recorded by the coaxial camera. The estimation procedure proposed in this section is based on several steps. Before going into the details, a brief schematic description of the complete algorithm is given, and the processing chain is depicted in Fig. 7.

The processing chain is composed of two main blocks, namely, the featuring and mapping blocks. The featuring block takes the raw signal as the input, i.e., images of process emission, and aims at synthesizing its information in a limited number of descriptors, called features. In the proposed procedure, three steps of featuring are accomplished: image analysis, processing of geometrical features in the time domain, and representation of their dispersion from a statistical point of view.

The mapping block takes the features as input and tries to map them onto the output variable. In other words, the mapping block aims at fitting a model between features and output. In the proposed algorithm, a neural network (NN) model is used. 


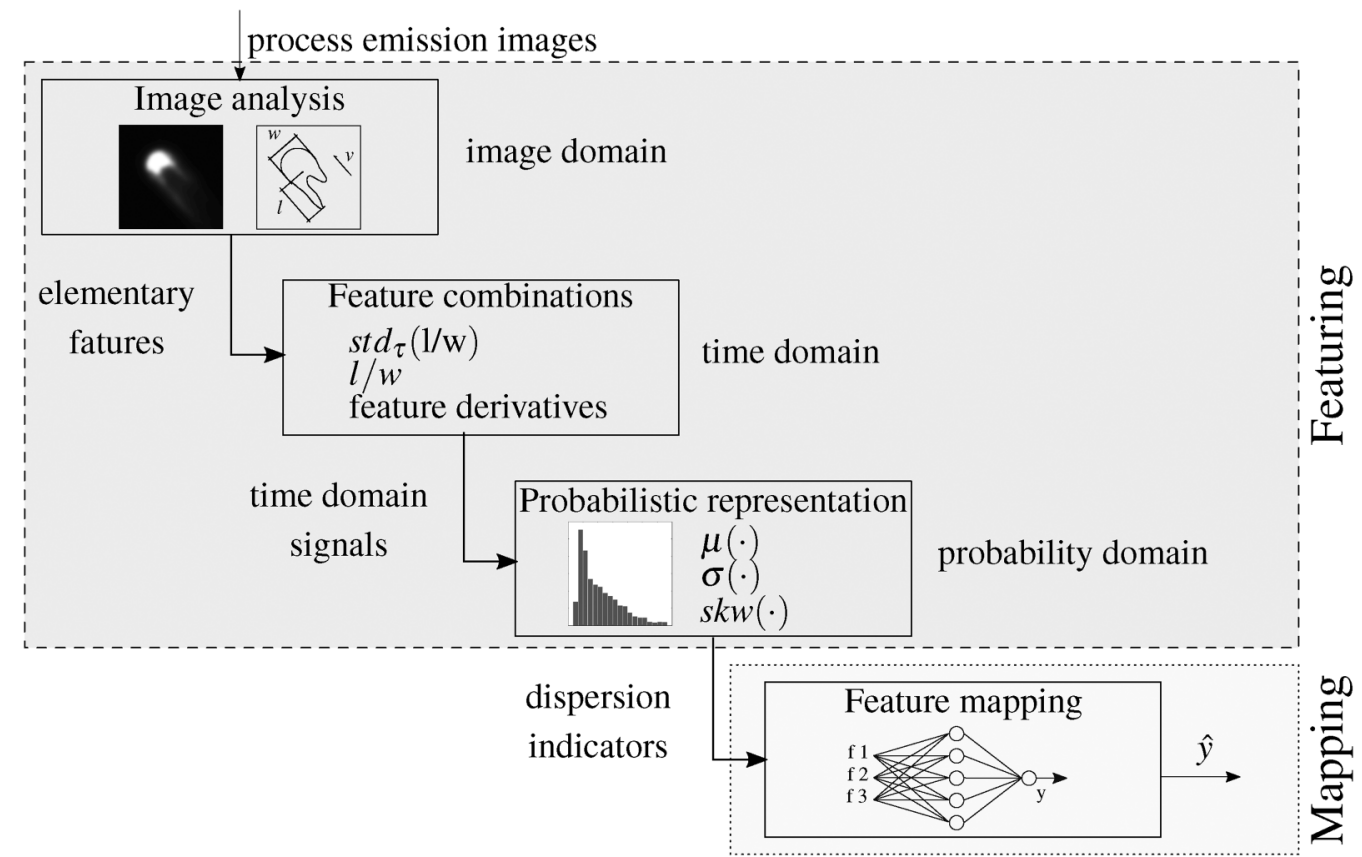

FIG. 7. Scheme of the machine-learning estimation algorithm. The information contained in process emission images is manipulated in multiple processing steps.

\section{A. Image analysis of process emission images}

The estimation procedure starts with the extraction of synthetic features of the laser irradiated zone from process images. An image is represented by a matrix $\Xi \in \mathbb{N}^{M \times N}$ with $M, N \in \mathbb{N}$ being the number of rows and columns, respectively. The matrix values, usually called gray values or gray levels, are bounded in the interval $g_{m, n} \in\left[0,2^{n b i t}-1\right]$, where nbit is the number of bits of the analog to digital conversion performed by the camera sensor and $m \in[0, M], n \in[0, N]$ are the matrix indexes. The algorithm is based on hard thresholding: image $\Xi$ is binarized according to a static threshold as

$$
\check{g}_{m, n}= \begin{cases}1 & g_{m, n} \geq C, \\ 0 & \text { otherwise }\end{cases}
$$

where $\check{g}_{m, n}$ is a pixel value of the binary image resulting from the operation and $C$ is the value of the threshold. The resulting binary image, $\Xi$, has the same size of $\Xi$ and is characterized by a set $B \subset$ $\check{\Xi} \mid \check{g}_{m, n}=1$ called blob of white points representative of points in $\Xi$ having values higher than $C$. This thresholding operation has a physical meaning. Considering a body emitting electromagnetic radiation, the value of $C$, i.e., a selected emission intensity, is related to the temperature $T_{C}$ at which the body is emitting. Thus, the selection of the value of $C$ and the thresholding operation permit to focus the attention to the phenomena that occurs at a (indirectly) selected temperature $T_{C}$. This eventually leads to the partial exploitation of the process emission images that can give further information considering other values of emission. The value of $C$ was set iteratively to emphasize the phenomena that are close to the melting temperature of steels. Since the two studied materials have a similar melting temperature, the value of $C$ has been maintained equal to 30 for both materials. According to the cutting direction, the following geometrical information are defined:

(1) blob centroid, $c$, i.e., the center of mass of the blob;

(2) blob width, $w$, i.e., the maximum dimension of the blob perpendicular to the cutting direction; and

(3) blob length, $l$, i.e., the maximum dimension of the blob parallel to the cutting direction extending from the centroid to the blob tail.

These quantities are calculated together with the image intensity, $I$, of $\Xi$ and constitute the elementary features by which a image is represented. These features were selected by visually inspecting the image shape variations for different dross attachment conditions. High-dross cuts are usually characterized by an elongated blob shape and by a greater variation in their length. Conversely, drossfree cuts are characterized by a stable and usually smaller blob shape. The details of the algorithm are now briefly explained. At first, image intensity is calculated as

$$
I=\sum_{m=1}^{M} \sum_{n=1}^{N} g_{m, n} .
$$


The blob centroid is then computed as

$$
c=\left(\begin{array}{c}
c_{x}=\frac{1}{N_{w}} \sum_{m=1}^{M} m \check{g}_{m, n} \\
c_{y}=\frac{1}{N_{w}} \sum_{n=1}^{N} n \check{g}_{m, n}
\end{array}\right),
$$

where $N_{w}$ is the total number of white points in the blob, given by

$$
N_{w}=\sum_{m=1}^{M} \sum_{n=1}^{N} \check{g}_{m, n} .
$$

For the calculation of $w$ and $l$, the lines passing through $c$ perpendicular and parallel to the cutting direction are considered. The cutting direction is derived from the cutting speed in $x$ and $y$ directions and transferred to the image reference frame through a rigid rotation. (Usually, in the considered cutting application, there is a fixed and constant angle of misalignment between the machine reference frame and the camera reference frame.) As is well known, the distance of the point $p_{0}=\left(x_{0}, y_{0}\right)$ from a line $\mathbf{r}=\{(x, y) \mid a x+$ $b y+c=0\}$ is equal to

$$
d=\frac{\left|a x_{0}+b y_{0}+c\right|}{\sqrt{a^{2}+b^{2}}} .
$$

If the absolute value is removed from the latter equation, the distance becomes positive for points above the line and negative for points below. This modification is useful in our case for calculating the width as the difference between the part of the blob that lies above and below the line. Indicating as $(p-\mathbf{r})$ the line to point distance with sign, the blob width is calculated as

$$
w=\underbrace{\max _{p}\left(p-\mathbf{r}_{\|}\right)}_{w_{+}}-\underbrace{\min _{p}\left(p-\mathbf{r}_{\|}\right)}_{w_{-}},
$$

where $p=(m, n)$ is the generic coordinate of $\check{g}_{m, n} \in B$ and $\mathbf{r}_{\|}$indicates the line parallel to the cutting direction passing through $c$.

The same applies for the calculation of the blob tails length, i.e., the distance between the blob centroid and the maximum blob tail extension. The positive and negative distances between the line perpendicular to $v$ passing through $c$ and each point of the blob are considered and one of them is selected according to the cutting direction, yielding

$$
l= \begin{cases}l_{+}=\max _{p}\left(p-\mathbf{r}_{\perp}\right) & v_{y}>0, \\ l_{-}=-\min _{p}\left(p-\mathbf{r}_{\perp}\right) & \text { otherwise, }\end{cases}
$$

where $v_{y}$ is the $y$-component of the cutting speed $v$. Notice that the condition in (12) depends by the experimental setup and the fixed orientation between the camera and the $x, y$ cutting directions.

To improve accuracy, the centroid position is averaged with a moving average among 20 frames. This is to avoid that especially variations in blob length are damped by a change in the centroid position and, therefore, not captured. An example of the application of the algorithm is given in Fig. 8. The computational time for processing one image with compiled code is approximately $0.2 \mathrm{~ms}$. As a result, real-time image analysis is feasible being the target

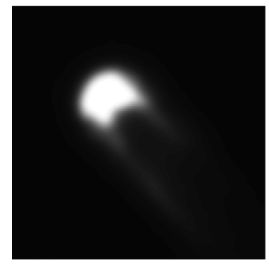

(a) Process emission image.

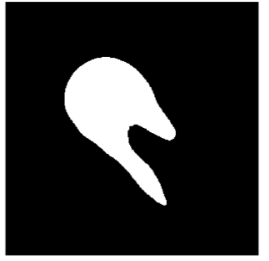
(b) Binarized image.

FIG. 8. Application of the image analysis algorithm for the extraction of geometrical features from process emission images (a); the steps of binarization (b) and the obtained features (c) are shown. The cutting direction is rotated by $45^{\circ}$ due to the relative orientation between the image and the axis reference frames.

image acquisition rate equal to $1500 \mathrm{fps}$ corresponding to a sampling period of approximately $0.67 \mathrm{~ms}$.

\section{B. Signal processing in the time domain}

The extraction of the geometrical information for a complete cut yields time signals that can be analyzed and combined to obtain other features. The image characteristic signals which reveal the form factor of the blob that are interesting for dross attachment estimation are three, namely, $w(k), l(k)$, and $I(k)$, respectively. To start, only quasisteady-state phenomena are considered, i.e., the portions of the cut geometry having constant process parameters. The transients due to the path corners are neglected as shown in Fig. 9. The final target is, therefore, to link quasisteady-state dross attachment formation with features in the same quasisteady-state locations.

From the three elementary features, the ratio between length and width of the blob, $l / w$, being the shape factor of the blob and its variation in the form of the moving standard deviation, $\operatorname{std}_{\tau}\left(\frac{l}{w}\right)$, are computed. Indeed, the dimensionless shape factor, $l / w$, and its

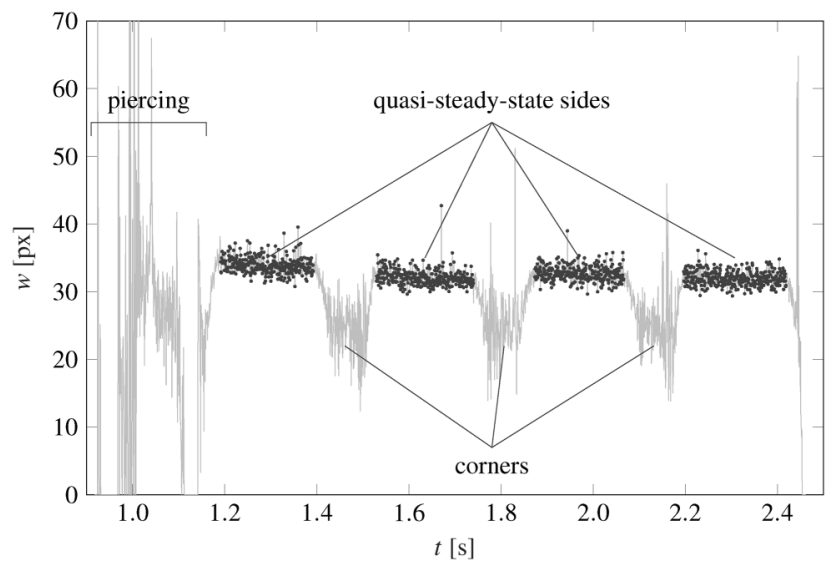

FIG. 9. Example of a time signal of width where different process phases are highlighted. The steady-state part only is considered for quality estimation. 
variation are considered more general and invariant parameters for the description of the blob shape with respect to the single dimensions. The moving standard deviation, $s t d_{\tau}(x(k))$, is calculated as

$$
\operatorname{std}_{\tau}(x(k))=\sqrt{\frac{\sum_{n=k-\tau+1}^{k}(x(n)-\bar{x})^{2}}{\tau-1}},
$$

where $x(k)$ was substituted by $l(k) / w(k)$ during the data processing. Finally, the time derivatives of the three elementary signals are also considered as possible features. The time derivatives have been computed as

$$
\frac{d x}{d t}(k)=\frac{x(k)-x(k-1)}{\Delta t} \quad k \geq 1,
$$

where $x(k)$ was substituted by $l(k), w(k), I(k)$, and $l(k) / w(k)$ during the data processing.

\section{Statistical representation of time-based features}

Instead of looking at point-to-point correlation between features and dross attachment, a stochastic characterization of the features themselves is proposed. Let us notice that in general time signals considered over a time interval, $\tau$, may be described as a sample distribution. These distributions are not easily described by conventional parametric ones; as a consequence, they are more compactly represented by their mean value and their second and third central moments. The definition of the $q$ th central moment for a real valued random variable $X$ is as

$$
\mu_{q}=E\left[(X-E[X])^{q}\right]=\int_{-\infty}^{+\infty}(x-\mu(x))^{q} f(x) d x,
$$

where $E$ indicates the expectation operator, $f(x)$ is the probability density function of $X$, and $\mu(x)$ is its the mean value. The first two moments, i.e., for $q=[0,1]$ are equal to 1 and 0 , respectively. The second central moment is usually called variance and denoted by $\sigma^{2}(x)$; finally, the third central moment is used to define the skewness of a distribution, i.e., a measure of its symmetry and denoted by $s k(x)$.

As a result, the total number of features candidate to be mapped onto process output is 27 , i.e., $3 \times 9: 3$ statistical indicators times the 9 features composed by the image analysis features, the two combinations and the four derivatives,

$$
\begin{gathered}
\mu(x), \sigma^{2}(x), s k(x), \\
x=\left\{w, l, I, l / w, \operatorname{std}_{\tau}(l / w), d l, d w, d I, d(l / w)\right\},
\end{gathered}
$$

where $d l, d w, d I$, and $d(l / w)$ denote the time derivatives $d l / d t, d w / d t, d I / d t$, and $d(l / w) / d t$.

\section{Feature mapping}

The goal of this section is the definition of a model between a limited number of features (modeled with their sample distribution) and the system output, $y$. In this study, an artificial neural network (ANN) is used as mapping tool and this choice is made based on the following rationale. ANNs do not require the formulation of a parametric model; complexity can be controlled by limiting the number of neurons and layers of the net to avoid over fitting. During the mapping phase, a model for each material thickness was developed. Indeed, relatively big differences are observed when comparing process emission images of different material thickness as reported in Fig. 10; this eventually leads to low accuracy and makes the use of a "global" model not satisfactory. To produce different models according to the plate thicknesses, the data acquired during the experiments were divided in subsets of approximately 20 specimens per set. These subsets contained a balanced number of different dross attachment conditions to correctly train the ANN-based model. It is, therefore, emphasized that the development of different models does not increase the required number of experiments and does not add significant complexity. This is due to the fact that the complexity of each model is the same and what changes is the value of the coefficients only. To express the prediction model in real-time conditions, a time window of size $\tau$ is used to evaluate the statistical representation of features over a sliding window. Note that, in a real-time estimation, the window-size has two main effects:

(1) during the initial part of the cut the estimation needs to reach its steady-state before being accurate and

(2) after convergence, the estimation is performed evaluating the data over a backward sliding window. The low-pass properties

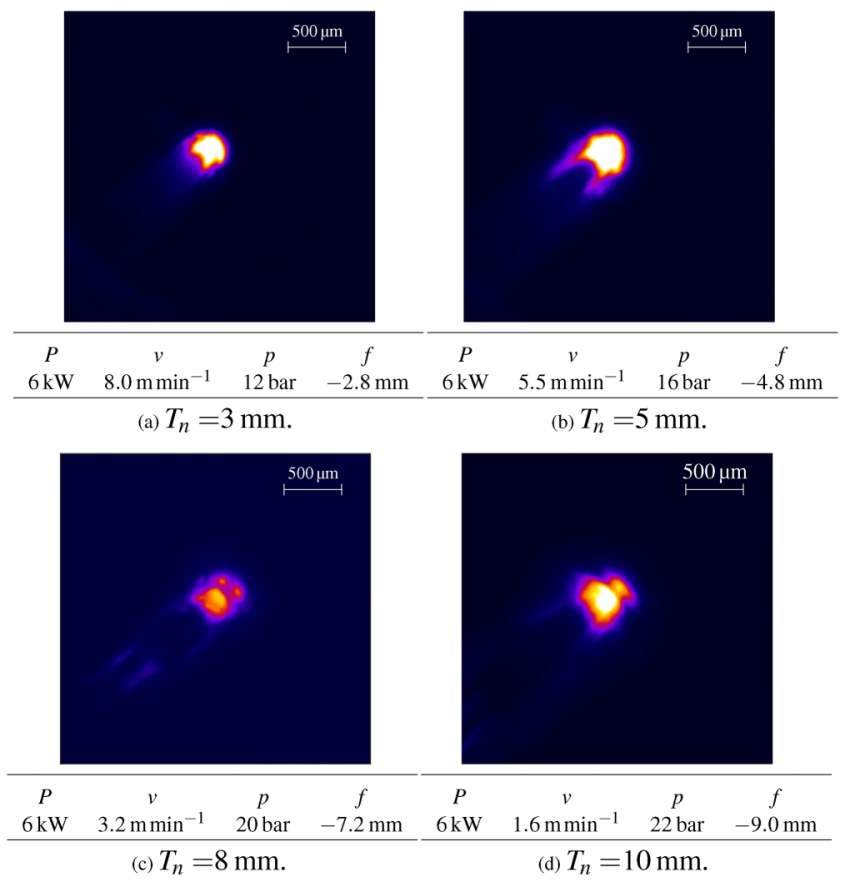

FIG. 10. Examples of process emission images of $X 5 \mathrm{CrNi18-10}$ for (a) 3 , (b) 5 , (c) 8 , and (d) $10 \mathrm{~mm}$ material thicknesses. The monitoring parameters are the same for all images and a transformation in pseudocolors is applied to facilitate the visualization; the color scale indicates the image intensity. 


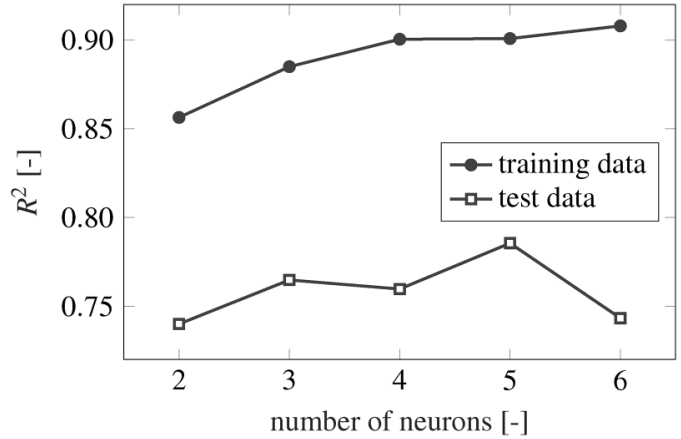

FIG. 11. $R^{2}$ parameter as a function of the number of neurons.

of the moving average filter introduce some filtering effect on the system dynamics of the cutting process.

As a consequence, the lower the $\tau$, the prompter the activation of the control system, and the more reduced the filtering effects introduced in the prediction dynamics. A shallow neural network structure having a single hidden layer was initially selected. Starting from the 27 candidates, the number of inputs of the $\mathrm{NN}$, the number of neurons of the hidden layer, and the window $\tau$ where selected iteratively by performing sensitivity analyses. The results of the sensitivity analysis are reported in Figs. 11 and 12, respectively. It is shown that the model accuracy does not increase significantly for a number of neurons higher than 5 and for a time window larger than $150 \mathrm{~ms}$. The two parameters were, thus, set accordingly to these two values, respectively. The number of input features was reduced to only three evaluating iteratively the most informative features' set and the final accuracy of the model that does not significantly increase for a larger number of features. As a results, the triplet composed of the mean values of blob tails length, $\mu(l)$, blob width, $\mu(w)$, and image intensity, $\mu(I)$ was selected as the most informative. During these analysis steps, 20 specimens of $3 \mathrm{~mm}$ and 20 specimens of $5 \mathrm{~mm}$ were used separately. Thanks to the

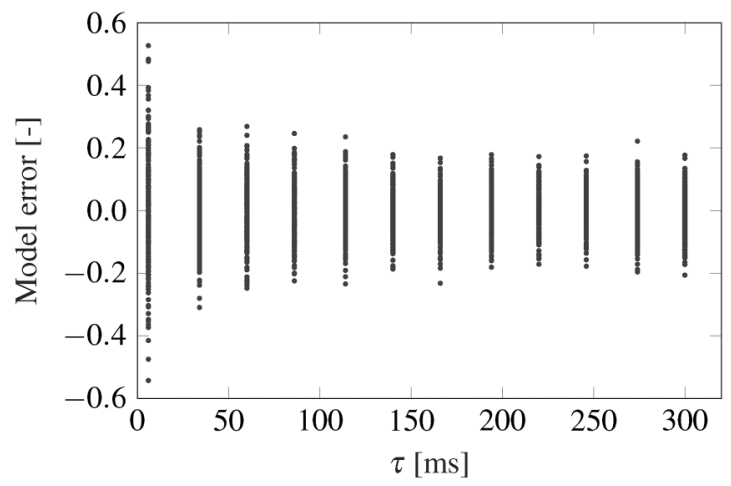

FIG. 12. Model error as a function of the window $\tau$. high time resolution of the camera, the two models rely on approximately 25000 and 35000 data points for 3 and $5 \mathrm{~mm}$, respectively. Finally, during the training phase of the ML algorithm in Sec. IV, the data set was split in the three sets for training, validation, and test with $80 \%, 10 \%$, and $10 \%$, respectively.

\section{DROSS ATTACHMENT ESTIMATION BY MEANS OF CONVOLUTIONAL NEURAL NETWORKS}

CNNs are usually employed in classification problems, i.e., problems where the target is to classify the data in some classes according to specific features. In our context, we define the classes as yes-dross and no-dross according to the binary quantity $h_{t h}^{2}(k)$ defined in (4). To compare the results obtained with $\mathrm{CNN}$ and $\mathrm{ML}$ algorithms, the continuous system output $\hat{y}_{C N N}$ is calculated as

$$
\hat{y}_{C N N}(k)=\frac{1}{\tau+1} \sum_{n=0}^{\tau} \hat{h}_{t h}^{2}(k-n),
$$

where $\hat{h}_{t h}^{2}$ is the estimate of $h_{t h}^{2}(k)$ obtained as output of the CNN model.

\section{A. CNN fundamentals}

CNNs are a type of ANNs. The CNN can automatically learn a hierarchy of features from the input image matrices, which prove to be better than those hand-crafted features extracted by carefully designed complex algorithms. ${ }^{39}$ CNNs automatically assign importance to various aspects and objects in the images. By discovering and differentiating those aspects and objects, CNNs can learn filters to isolate a specific characteristic inside images. A typical $\mathrm{CNN}$ architecture consists of several nested convolutional and pooling layers followed by fully connected layers at the end. A compact presentation of this kind of network [Input-Conv-ReLUPool-FC] consists of the following five layers:

- Input: the inputs of the $\mathrm{CNN}$ are $210 \times 210$ pixel 1 -channel grayscale images, containing the intensity values of each pixel.

- Conv: the convolutional layers apply over the whole input image a set of learned filters of small size (like $3 \times 3$ or $5 \times 5$ ), updated during training, each of which is connected to only a small region of the output. The convolution operation is applied over the whole input image, performing a matrix multiplication between the filter and the portion of the image over which the kernel is hovering. Some numerical parameters such as the number of filters, the size and the architecture of the whole network need to be specified.

- ReLU: ReLU (rectified linear units) is the activation function used to adding nonlinear transformations to the output of the convolutional or fully connected layers. The function is $f(x)=$ $\max (0, x)$ and it replaces all negative pixel values with a zero. Convolution is a linear operation since it applies an element-wise matrix multiplication and additions. Introducing nonlinearity in the $\mathrm{CNN}$ accounts for most of the real-world data that contain nonlinearity, otherwise unrecognizable by the convolution operation itself.

- Pool: the pooling layer performs a form of nonlinear downsampling along both spatial dimensions, leading to reduced 
spatial size of the convolved features. It aims to reduce the amount of the network parameters and the computational cost, keeping the most important information inside the convolved features. Extracting dominant features results in convolved features which are rotational and positional invariant. Simple ANN have little invariance to shifting, scaling, and other forms of distortion because they process images directly with pixel values as inputs. Pooling layers allow $\mathrm{CNN}$ to be almost completely invariant to forms of distortion.

- FC: All the neurons in the fully connected layers are connected to all the units of the last layer. And, the last fully connected layer generates the output of the whole network.

These layers are stacked together to form a CNN, the input is fed forward into the network, and the hyperparameters are updated by the backpropagation algorithm.

\section{B. Structure of the CNN for dross attachment estimation}

As shown in Fig. 13, the custom CNN structure used consists of 24 layers. To summarize, the architecture can be described as input layer, $C(32,3,3), S(2,2,2), C(32,3,3), C(64,3,3), \quad S(2,2,2)$, $C(128,3,3), C(128,3,3), S(2,2,2), F C(1024), F C(1024), F C(2)$, and softmax layer. $C(n, 3,3)$ represents a convolutional layer with $n$ filters of kernel size $3 \times 3, S(2,2,2)$ represents a pooling layer with a subsampling factor of $2 \times 2$ by stride 2 in both dimensions, and $F C(n)$ represents a fully connected layer with $n$ neurons. The pooling strategy adopted in all the pooling layers is max-pooling, which is robust to distortions. After all the convolutional layers, a ReLU layer is applied. The last fully connected layer generates the output, composed by two numbers, corresponding to the classes adopted (dross present or not), meaning the estimation of each image's class membership probability. Softmax layer is used to select the class with higher probability.

Images show the real-time emission of the laser irradiated zone, as described in Sec. II. Since images are captured from a fusion-cutting process of stainless steel (X5CrNi18-10) and mild steel with different thicknesses $(3,5,8$, and $10 \mathrm{~mm}$ ), we want to achieve a general purpose recognition of dross: the $\mathrm{CNN}$ is trained to recognize defected images for the specimen of both materials and the different thicknesses. As can be seen in Fig. 10, different cuts generate different blobs in the images because different thickness produces different cutting process. Our $\mathrm{CNN}$ is trained to recognize defect in different kind of cutting processes, as our goal is to achieve a general purpose dross recognition.

\section{CNN training and testing}

During training, the whole data set is divided into three different subsets:

- training set, used during training to update weights and biases of $\mathrm{CNN}$;

- validation set, used for validating updates during training; and

- test set, used for testing accuracy results with $\mathrm{CNN}$ after the whole training procedure is finished.

In our experiments, we randomly choose $70 \%$ of the data as the training set, $20 \%$ of the data as the validation set, and the rest $10 \%$ as the test set. Due to the high complexity of the model if compared to the one in Sec. IV D, the full data set comprehensive of all material thicknesses was used. In fact, the number of numerical parameters to be set during the training phase is much higher and the model requires more data to achieve a good accuracy. As mentioned, the output of the trained CNN is the binary estimate $\hat{h}_{t h}^{2}(k)$ of $h_{t h}^{2}(k)$. First training sessions showed that image and dross profile $h^{2}(k)$ frequencies were decoupled; the final accuracy results were always around the "random guess" percentage. This means that $\mathrm{CNN}$ could not learn from original images as-is probably because of a too demanding requirement of point-to-point correlation. Analyzing the spectrum of $h^{2}(k)$, we discovered that the maximum informative frequency was equal to $150 \mathrm{~Hz}$. For this reason, we introduced a preprocessing averaging operation creating a second data set where ten subsequent images are averaged pixel-by-pixel. This second averaged data set has a reduced frequency of approximately $150 \mathrm{~Hz}$ as the dross attachment signal. As a result, a deep knowledge of the laser cutting process is required to correctly train the $\mathrm{CNN}$.

\section{Training results and misclassification issues}

Training results on the averaged data set show a final accuracy of $93 \%$ over the validation set and a final accuracy of $92 \%$ over the test set. This means there is no observation of over-fitting in the training process. A misclassification of $7 \%-8 \%$ is acceptable in this application field because our $\mathrm{CNN}$ is trained to recognize defect in a relatively wide process window. Images of different materials and thicknesses are quite different, so the $\mathrm{CNN}$ has to learn a wide set

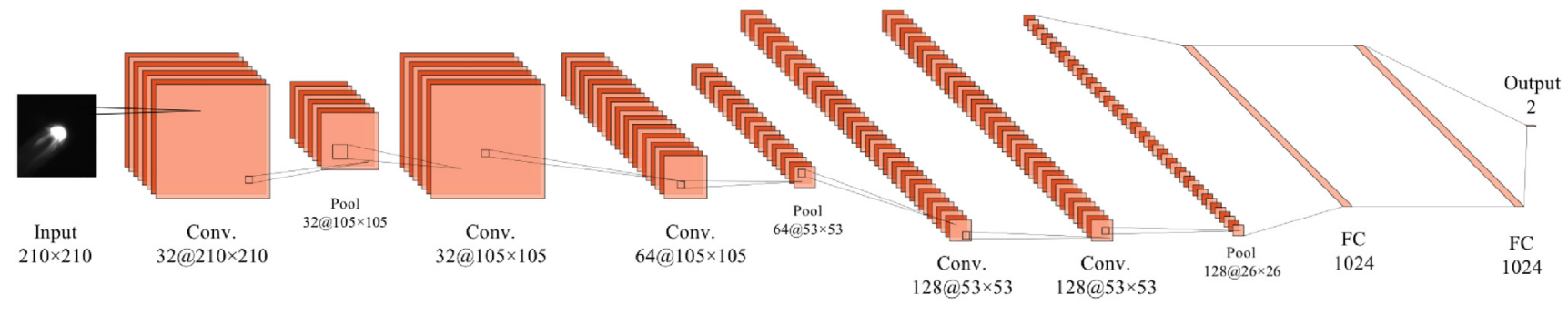

FIG. 13. Schematic architecture of the custom CNN. 
of features to classify them correctly. On the other hand, we decided to understand and investigate why there is such misclassifications. In particular, we aimed to understand if there exists particular conditions of material and/or thicknesses related to misclassification. It turns out that there was an even distribution of misclassifications over thickness and materials, meaning that characteristics of specimen do not influence training results and misclassification.

Overall, the performances are satisfying. More precisely, dross attachment estimation is perfectly accurate in dross-free cuts and in cuts with very high-dross cuts, that means the error on defects estimation is approximately zero. An accurate analysis of cuts with intermediate-dross values shows that high-frequency changes in $h^{2}(k)$ lead to a wrong classification with the CNN. In particular, when $h^{2}(k)$ is around the chosen threshold and switches frequently above and below it, the CNN-based classification contains a nonnegligible number of false positives.

\section{ESTIMATION RESULTS AND ALGORITHM COMPARISONS}

The results of the two estimation approaches are now shown and compared. Two models ML models for 3 and $5 \mathrm{~mm}$ that rely on approximately 25000 and 35000 data points, respectively, are compared to the results of the $\mathrm{CNN}$ model for the same cuts. We selected these two thickness values among others because they represents the most challenging examples that have been investigated. In fact, these reported examples are close to the industrial field reporting reasonable and low dross attachment levels, thus making the estimation more challenging. Figures 14 and 15 show the estimation results for $3 \mathrm{~mm}$ specimens. The images show the two estimates $\hat{y}_{M L}$ and $\hat{y}_{C N N}$ of the two algorithms explained in Secs. IV
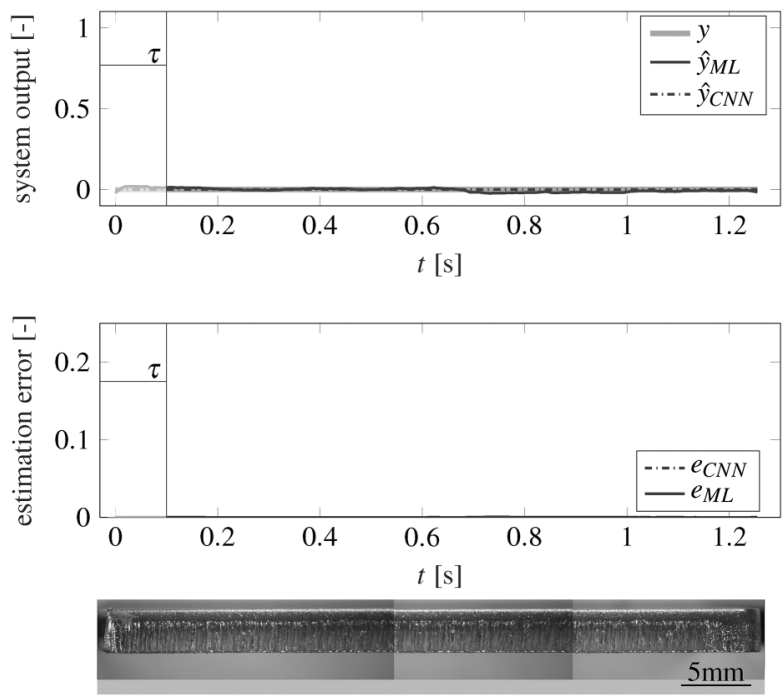

FIG. 14. System output prediction for a dross-free cut of stainless steel $\mathrm{X} 5 \mathrm{CrNi18}-10$ of $3 \mathrm{~mm}\left(P=6 \mathrm{~kW}, f=-2.8 \mathrm{~mm}, v=7 \mathrm{~m} \mathrm{~min}^{-1}, p=12 \mathrm{bar}\right)$.
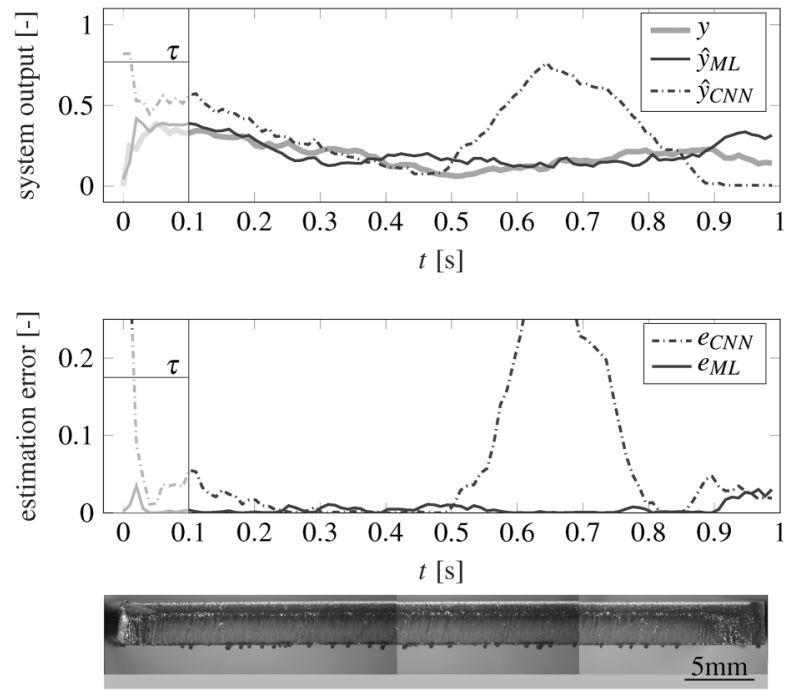

FIG. 15. System output prediction for a high-dross cut of stainless steel $X 5 \mathrm{CrNi18-10}$ of $3 \mathrm{~mm} \quad\left(P=6 \mathrm{~kW}, \quad f=-2.8 \mathrm{~mm}, \quad v=9.7 \mathrm{~m} \mathrm{~min}^{-1}\right.$, $p=12$ bar)

and $V$, respectively. The estimation errors are computed as

$$
e_{i}=\left|y-\hat{y}_{i}\right| \quad i=\{M L, C N N\} .
$$

One may note that for dross-free cuts, the two algorithms yield similar performance. For intermediate and high dross attachment
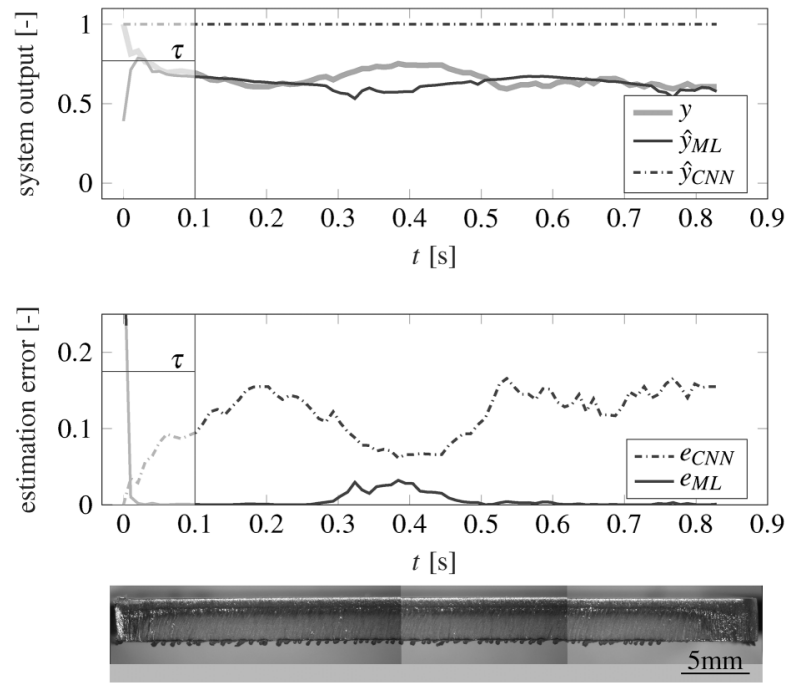

FIG. 16. System output prediction for a intermediate-dross cut of stainless steel X5CrNi18-10 of $3 \mathrm{~mm} \quad\left(P=6 \mathrm{~kW}, \quad f=-2.8 \mathrm{~mm}, \quad v=8.5 \mathrm{~m} \mathrm{~min}^{-1}\right.$, $p=12$ bar) 

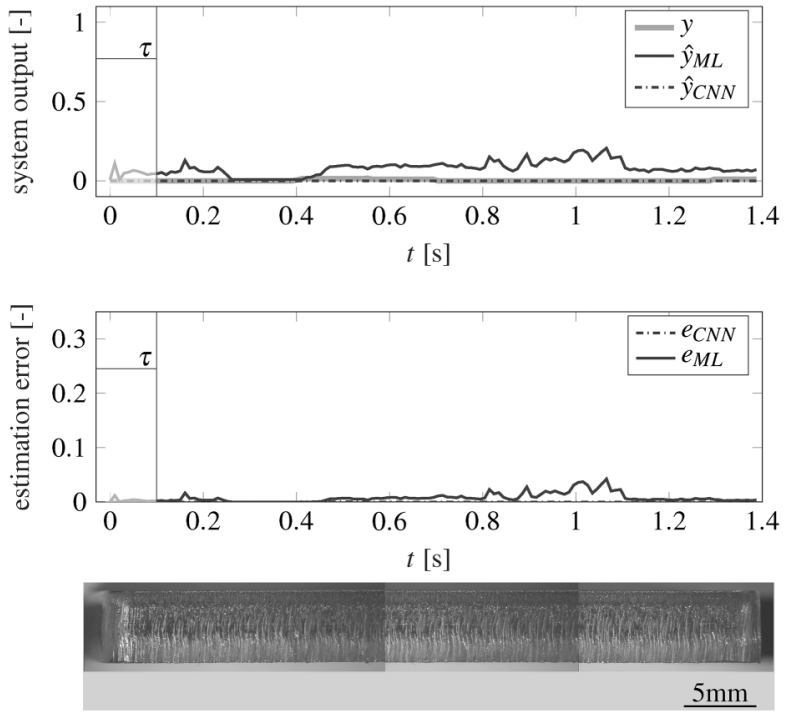

FIG. 17. System output prediction for a dross-free cut of stainless steel X5CrNi18-10 of $5 \mathrm{~mm} \quad\left(P=6 \mathrm{~kW}, \quad f=-4.3 \mathrm{~mm}, \quad v=6.1 \mathrm{~m} \mathrm{~min}^{-1}\right.$, $p=16$ bar $)$.

conditions, the estimation error of both models slightly increases but still remains really low. Furthermore, the ML algorithm slightly outperforms the CNN model.

Figures 17-19 show the estimation results for $5 \mathrm{~mm}$ specimens. Both models yield good performance and the model error is
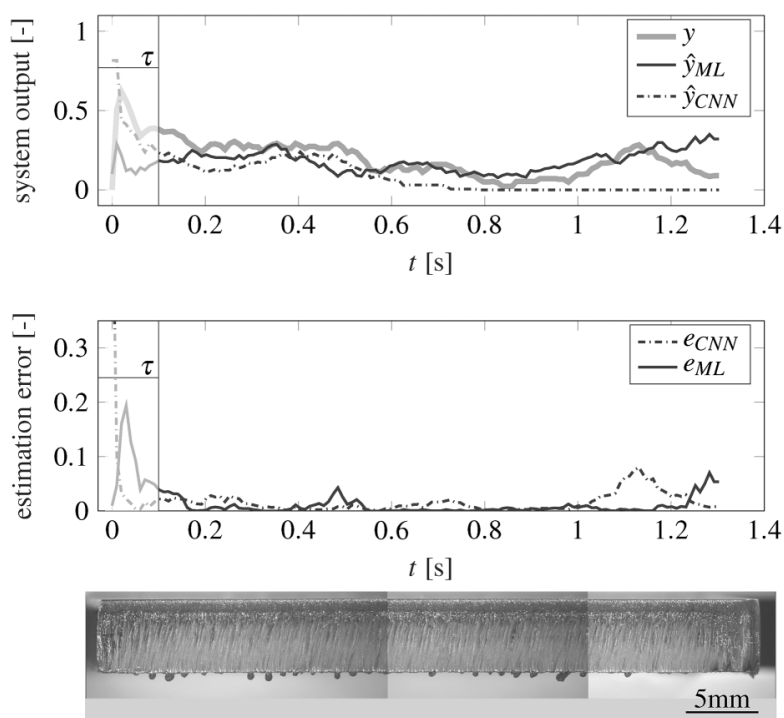

FIG. 18. System output prediction for a low-dross cut of stainless steel X5CrNi18-10 of $5 \mathrm{~mm} \quad\left(P=6 \mathrm{~kW}, \quad f=-4.3 \mathrm{~mm}, \quad v=6.5 \mathrm{~m} \mathrm{~min}^{-1}\right.$ $p=12$ bar).
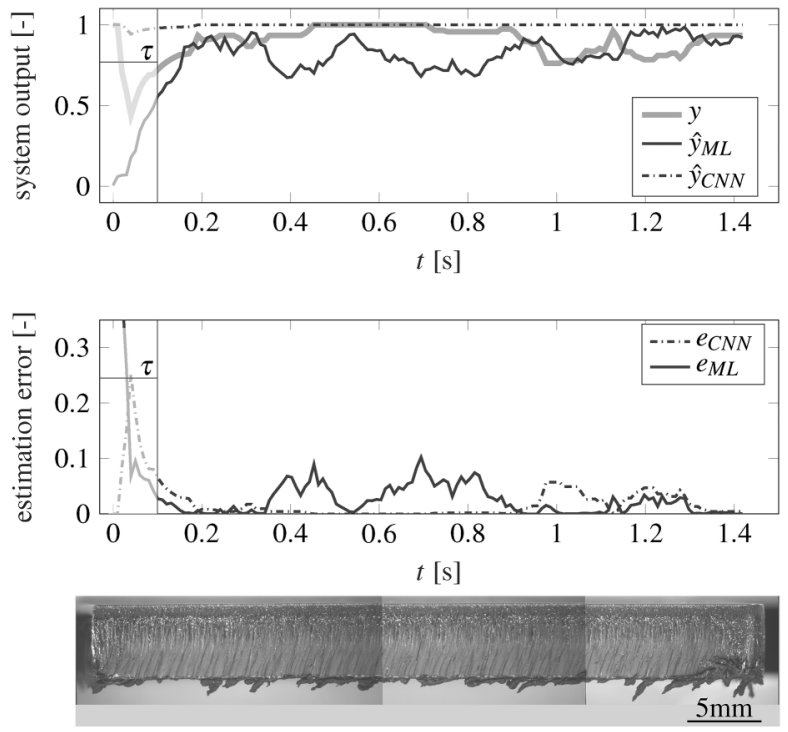

FIG. 19. System output prediction for a high-dross cut of stainless steel X5CrNi18-10 of $5 \mathrm{~mm}\left(P=6 \mathrm{~kW}, f=-3.3 \mathrm{~mm}, v=6 \mathrm{~m} \mathrm{~min}^{-1}, p=8 \mathrm{bar}\right)$.

almost always lower than 0.1 . In this case, the $\mathrm{CNN}$ model performs slightly better than the ML model especially for dross-free and high-dross cuts. For both models, it is observed that what generally is addressed as a stationary level of dross is described by significant fluctuations in the $y$ signal (e.g., Figs. 16 and 18). In other words, the resolution of the proposed approach is higher than the requested level of accuracy. On the one side, this gives the possibility to finely adjust process parameters to reach the desired quality. On the other side, these fluctuations might be traced by a closed-loop controller leading to an unnecessary fast parameters change. This behavior will need to be considered when tuning and defining the controller structure.

Finally, the two algorithms are compared in terms of computational cost in Table III. The big difference between the two computational times is mainly due to the low computational cost of the image analysis algorithm in Sec. IV A that requires a loop on all element of the image only. In the $\mathrm{CNN}$ algorithm instead, the relatively high number of convolutional layers increases significantly the required computational effort. Considering the high acquisition rate of $1500 \mathrm{fps}$, the $\mathrm{CNN}$ algorithm would not permit a real-time application. The ML algorithm is in this concern more promising even if the margin of approximately $0.35 \mathrm{~ms}$ may not be sufficient for a reliable image acquisition and transfer from the camera to the PC. Future works will deeply address this issue.

TABLE III. Average computational times for the two algorithms.

\begin{tabular}{cc}
\hline \hline Algorithm & Computational time (ms) \\
\hline ML & 0.31 \\
$\mathrm{CNN}$ & 8.43 \\
\hline
\end{tabular}




\section{CONCLUDING REMARKS}

In this study, two algorithms for the real-time, continuous estimation of the dross attachment produced during the laser fusion-cutting process have been presented. Both algorithms are based on the information collected by a coaxial camera, and their performance are assessed based on a reference dross signal measurement appropriately defined and computed. The process emission images are analyzed differently in the two algorithms: in the ML algorithm, geometrical features are extracted from images and processed to obtain their probabilistic representation, finally mapped with a shallow neural network onto the system output. In the $\mathrm{CNN}$ algorithm, instead, a direct mapping from images to the system output is performed and the featuring phase is delegated to the convolutional layers of the CNN. Experimental results prove that both algorithms can be used to accurately estimate the system output and, therefore, the amount of dross attachment of real cuts. The ML algorithm is deemed more promising for real-time applications thanks to its extremely low computational time. Current work is being focused on the development of a closed-loop control algorithm to adapt process parameters to obtain a continuous regulation of the dross attachment according to a desired value. Some aspects contained in the presented paper are currently part of a pending patent application.

\section{ACKNOWLEDGMENTS}

The authors are grateful to the BLM GROUP for the great cooperation and, in particular, to the colleagues of Adige S.P.A. for the professional support received during the study and the precious hints about the laser cutting process. The project presented in this paper has been funded with the contribution of the Autonomous Province of Trento, Italy, through the Regional Law No. 6/99. Name of the granted project: LT4.0.

\section{APPENDIX: COMPONENTS OF THE EXPERIMENTAL SETUP}

The utilized laser cutting machine is a LC5 (Adige-SYS S.p.A. BLMGROUP, Levico Terme, Italy) endowed with a YLS-6000-CUT fiber laser source (IPG Photonics Coorp., Oxford, Massachusetts) and a HPSSL cutting head (Precitec GmbH \& Co., Gaggenau, Germany). The selected camera is a MQ013MG-ON (Ximea $\mathrm{GmbH}$, Muenster, Germany) filtered with a 750FS00-25 (Andover Corporation, Salem, New Hampshire). The microcontroller is a STM32F746ZG (STMicroelectronics, Geneve, Swiss). Finally, the code for image processing was implemented in MATLAB and run on a Dell XPS 15 in. equipped with a Intel i7 (Gen 7) processor.

\section{REFERENCES}

1D. Belforte, "2018 was another record year, contending with turmoil," Ind. Laser Solutions 34, 9-12 (2019).

${ }^{2}$ C. L. Caristan, Laser Cutting Guide for Manufacturing (Society of Manufacturing Engineers, Dearborn, MI, 2004).

${ }^{3}$ W. M. Steen and J. Mazumder, Laser Material Processing, 4th ed. (Springer, London, 2010).

${ }^{4}$ J. Pocorni, A. F. H. Kaplan, D. Petring, and J. Powell, LIA Guide to High Power Laser Cutting (Laser Institute of America, Orlando, FL, 2017).
${ }^{\mathbf{5}}$ See https://www.blmgroup.com for more information about BLM GROUP (last accessed February 8, 2020).

${ }^{\mathbf{6}}$ See https://www.trumpf.com for more information about TRUMPF GmbH (last accessed February 8, 2020).

${ }^{7}$ M. Pacher, L. Monguzzi, L. Bortolotti, M. Sbetti, and B. Previtali, "Quantitative identification of laser cutting quality relying on visual information," in LiM 2017 Proceedings (German Scientific Laser Society, Munich, 2017), pp. 1-11.

${ }^{8}$ C. Goppold, T. Pinder, and P. Herwig, "Transient beam oscillation with a highly dynamic scanner for laser beam fusion cutting," Adv. Opt. Technol. 5, 61-70 (2016).

${ }^{9}$ F. O. H. Jorgensen, "Process monitoring during $\mathrm{CO}_{2}$ laser cutting," SPIE Proc. 1412, 198-208 (1991).

${ }^{10}$ P. Sforza and V. Santacesaria, " $\mathrm{CO}_{2}$ laser cutting: Analytical dependence of the roughness of the cut edge on the experimental parameters and process monitoring," SPIE Proc. 2207, 836-847 (1994).

${ }^{11}$ P. Sforza, M. Dell'Erba, V. Santacesaria, D. de Blasiis, and V. Lombardo, "A three-modules sensor for $\mathrm{CO}_{2}$ laser welding and cutting processes," SPIE Proc. 3097, 97-107 (1997).

12I. Decker, H. Heyn, D. Martinen, and H. Wohlfahrt, "Process monitoring in laser beam cutting on its way to industrial application," SPIE Proc. 3097, 29-37 (1997).

${ }^{13}$ H. Kaebernick, A. Jeromin, and P. Mathew, "Adaptive control for laser cutting using striation frequency analysis,” CIRP Ann. Manuf. Technol. 47, 137-140 (1998).

${ }^{14}$ J. De Keuster, J. R. Duflou, and J. P. Kruth, "Monitoring of high-power $\mathrm{CO}_{2}$ laser cutting by means of an acoustic microphone and photodiodes," Int. J. Adv. Manuf. Technol. 35, 115-126 (2006).

${ }^{15}$ J. De Keuster, J. R. Duflou, and J. P. Kruth, "Real-time adaptive control and optimisation of high-power $\mathrm{CO}_{2}$ laser cutting using photodiodes," in Proceedings of the 5th Lane Conference (Elsevier, Erlangen, 2007), p. 14.

${ }^{16}$ D. You, X. Gao, and S. Katayama, "Review of laser welding monitoring," Sci. Technol. Weld. Joining 19, 181-201 (2014).

${ }^{17}$ T. Purtonen, A. Kalliosaari, and A. Salminen, "Monitoring and adaptive control of laser processes," in Proceedings of the 4th Lane Conference (Elsevier, Furth, 2014), Vol. 56, pp. 1218-1231.

${ }^{18}$ N. Levichev, G. Costa Rodrigues, R. Dewil, and J. R. Duflou, “Anticipating heat accumulation in laser oxygen cutting of thick metal plates," J. Laser Appl. 32, 022018 (2020).

${ }^{19}$ B. Adelmann, B. Neumeier, M. Schleier, E. Wilmann, and R. Hellmann, "Optical cutting tear detection system for industrial fiber laser based cutting machines," in LiM 2015 Proceedings (German Scientific Laser Society, Monaco di Baviera, 2015).

${ }^{20}$ B. Adelmann, M. Schleier, B. Neumeier, E. Wilmann, and R. Hellmann, "Optical cutting interruption sensor for fiber lasers," Appl. Sci. 5, 544-554 (2015).

${ }^{\mathbf{2 1}}$ B. Adelmann, M. Schleier, B. Neumeier, and R. Hellmann, "Photodiode-based cutting interruption sensor for near-infrared lasers," Appl. Opt. 55, 1772-1778 (2016).

${ }^{22}$ M. Schleier, B. Adelmann, C. Esen, and R. Hellmann, "Cross-correlation-based algorithm for monitoring laser cutting with high-power fiber lasers," IEEE Sens. J. 18, 1585-1590 (2018).

${ }^{23}$ M. Schleier, B. Adelmann, B. Neumeier, and R. Hellmann, "Burr formation detector for fiber laser cutting based on a photodiode sensor system," Opt. Laser Technol. 96, 13-17 (2017).

${ }^{\mathbf{2 4}}$ M. Y. Huang and C. R. Chatwin, "A knowledge-based adaptive-control environment for an industrial laser cutting system,” Opt. Lasers Eng. 21, 273-295 (1994).

${ }^{25}$ C. Alippi, V. Bono, V. Piuri, and F. Scotti, "Toward real-time quality analysis measurement of metal laser cutting," in VIMS 2002: IEEE International Symposium on Virtual And Intelligent Measurement Systems (IEEE, Girdwood, 2002), pp. 39-44.

${ }^{26} \mathrm{P}$. Wen, Y. Zhang, and W. Chen, "Quality detection and control during laser cutting progress with coaxial visual monitoring," J. Laser Appl. 24, 032006 (2012). 
${ }^{27} \mathrm{Z}$. Hu and X. Yuan, "A machine vision detection algorithm for laser-cutting spark jet angle,” J. Intell. Fuzzy Syst. 37, 4595-4603 (2019).

${ }^{\mathbf{2 8}} \mathrm{H}$. Haferkamp, M. Goede, and A. von Busse, "Quality monitoring and assurance for laser beam cutting using a thermographic process control," SPIE Proc. 3824, 383-391 (1999).

${ }^{29}$ R. Poprawe, "Modeling, monitoring and control in high quality laser cutting," CIRP Ann. Manuf. Technol. 50, 12-15 (2001).

${ }^{30}$ J. R. Duflou, E. F. Sichani, J. D. Keuster, and J.-P. Kruth, "Development of a real time monitoring and adaptive control system for laser flame cutting," in International Congress of Applications of Lasers \& Electro-Optics (Laser Institute of America, Orlando, 2009), Vol. 2009, pp. 527-536.

${ }^{31}$ E. F. Sichani, J. De Keuster, J. P. Kruth, and J. R. Duflou, "Monitoring and adaptive control of $\mathrm{CO}_{2}$ laser flame cutting," in Physics Procedia Laser Assisted Net Shape Engineering 6, Proceedings LANE 2010 (Erlangen, Germany, 2010), Pt. 2, Vol. 5, pp. 483-492.

${ }^{32}$ E. Fallahi Sichani, S. Kohl, and J. R. Duflou, "Plasma detection and control requirements for $\mathrm{CO}_{2}$ laser cutting," CIRP Ann. 62, 215-218 (2013).

${ }^{33}$ L. Mazzoleni, A. G. Demir, L. Caprio, M. Pacher, and B. Previtali, "Real-time observation of melt pool in selective laser melting: Spatial, temporal and wavelength resolution criteria," IEEE Trans. Instrum. Meas. 69, 1179-1190 (2019).

${ }^{34}$ M. Pacher, L. Mazzoleni, L. Caprio, A. G. Demir, and B. Previtali, "Estimation of melt pool size by complementary use of external illumination and process emission in coaxial monitoring of selective laser melting," J. Laser Appl. 31, 022305 (2019).

${ }^{35}$ VDI-Gesellschaft Produktionstechnik, "Quality of cut faces of (sheet) metal parts after cutting, blanking, trimming or piercing - Laser cutting," Standard (Verein Deutscher Ingenieure, Duesseldorf, DE, 1994).

${ }^{36}$ C. Goppold, F. Urlau, T. Pinder, P. Herwig, and A. F. Lasagni, "Experimental investigation of cutting performance for different material compositions of $\mathrm{Cr} / \mathrm{Ni}$-steel with $1 \mu \mathrm{m}$ laser radiation," J. Laser Appl. 30, 031501 (2018).

${ }^{37} \mathrm{D}$. Lowe, "Object recognition from local scale-invariant features," in Proceedings of the Seventh IEEE International Conference on Computer Vision (IEEE, Kerkyra, 1999), Vol. 2, pp. 1150-1157.

${ }^{38}$ J. Canny, "A computational approach to edge detection," IEEE Trans. Pattern Anal. Mach. Intell. PAMI-8, 679-698 (1986).

${ }^{39}$ T. Wang, Y. Chen, M. Qiao, and H. Snoussi, "A fast and robust convolutional neural network-based defect detection model in product quality control," Int. J. Adv. Manuf. Technol. 94, 3465-3471 (2018). 